\title{
Recent changes to Pain Definition and Pain Classification
}

\author{
Takahiro Ushida*, Shyuhei Nagai, Hiroki Igari, Yuki Terajima and Keiko Owari \\ Multidisciplinary Pain Center, Aichi Medical University Nagakute, Japan
}

Submission: September 28, 2021; Published: October 08, 2021

*Corresponding author: Takahiro Ushida, Multidisciplinary Pain Center, Aichi Medical University Nagakute, Japan

\begin{abstract}
Pain is a phenomenon that we all experience in our daily lives, and it also provides information for detecting and responding to the bodily crisis. On the other hand, the nature and duration of pain vary, and there are significant individual differences. Therefore, it is necessary to classify pain based on neuroscience mechanisms and symptomatology and to provide appropriate treatment. In relation to these issues, the definition of pain has been revised in 2020 , and new concepts have been proposed, such as "Nociplastic Pain," the third mechanism of pain, and the classification of chronic pain in ICD11.
\end{abstract}

Meaning of pain to the organism and definition of pain

All people experience various types of pain in their daily lives from an early age. Pain is the most common symptom in many of diseases and injuries and always the first reason for hospital visits. As for why the pain perception system has developed in humans and animals, it is thought to be part of the system that allows each species to detect life-threatening situations as quickly as possible and take action to protect the body. In addition, pain is not only a means for organisms to detect and avoid crises, but it also triggers the immune system, autonomic nervous system, hormones, and other biological defense responses to injury and illness, which in turn are related to mental conditions. On the other hand, in chronic pain conditions, pain no longer plays a role in biological defense, and the pain avoidance behaviors exhibited by the patient may lead to disuse of the body or to pain behaviors that lead to conditions that reward the patient in society and at home. As such, the pain has various roles and aspects, and since ancient Egypt, Greece, and Rome, there has been debate about whether "pain" is sensation or an emotion.

This debate continued into the $20^{\text {th }}$ century, but in 1979 the International Association for the Study of Pain issued a definition of pain as "an unpleasant sensation, an emotional experience that is associated with a real or potential disturbance of the tissues or described in terms of such a disturbance." This definition was groundbreaking because it showed that pain is not only a sensation but also an emotional experience. This definition has been widely disseminated throughout the world until recent times and has been used by many international groups and organizations, including the World Health Organization (WHO). However, the old definition was revamped because it neglected the diversity of mind-body interactions, did not adequately address the pain of non-verbal populations such as newborns, the elderly, and nonhuman animals, and was not compatible with the latest brain science and psychosocial science [1]. The new definition of pain, revised in 2020, has been modified to "an unpleasant sensory and emotional experience associated with or resembling actual or potential tissue damage" [2]. This broadened the scope of coverage to include pain experienced by people and animals without verbal or social capacity. In addition, the pain was previously defined from the perspective of a medical professional, but with thanks to the new definition, it can now be defined from the perspective of the person experiencing pain. In addition, since pain is affected by an individual's past experiences and social circumstances, the following appendix is included to clarify '"pain'.

\section{Revised Pain definition (Raja et al. 2000)}

An unpleasant sensory and emotional experience associated with or resembling actual or potential tissue damage.

Note

$>$ Pain is always a personal experience that is influenced to varying degrees by biological, psychological, and social factors.

$>$ Pain and nociception are different phenomena. Pain 
cannot be inferred solely from activity in sensory neurons.

$>$ Through their life experiences, individuals learn the concept of pain.

$>$ A person's report of an experience as pain should be respected.

> Although pain usually serves an adaptive role, it may have adverse effects on function and social and psychological well-being.

> Verbal description is only one of several behaviors to express pain; inability to communicate does not negate the possibility that a human or a nonhuman animal experiences pain.

It is hoped that the revised definition will change the way pain is perceived and improve the perception and response to pain in actual clinical practice and society in many areas.

\section{Classification of pain}

As mentioned in the previous section, pain is influenced by various factors. There are temporal classifications, classifications based on the neuroscientific mechanism of pain generation, and the International Classification of Diseases created by the World Health Organization.

\section{Classification by duration of pain: acute pain and chronic pain}

In general, pain occurs when tissue is damaged due to injury, but the pain improves as the injured area heals. These are called acute pains, and as mentioned earlier, this type of pain has a role in crisis avoidance, but it also has a role in triggering reflexes and immune responses to improve the tissue. Prolonged pain (chronic pain), on the other hand, is defined as "pain that lasts more than three months". In this type of pain, anxiety, depression, or fear may occur due to the persistence of the pain, and behavioral changes may include treatment dependence, as well as avoidance of the fear associated with the pain. It has been reported that in $95 \%$ of cases of chronic pain, a psychiatric diagnosis will be given.

In many cases, there are psychological and social factors behind the persistence of a complex pain condition, and factors other than individual problems, such as exacerbation of the pain, are also involved. Chronic pain is also complicated by musculoskeletal injuries and nervous system disorders with irreversible and untreatable damage. Diagnosis is important in the treatment of chronic pain, and it is necessary to conduct psychosocial analysis as well as biological analysis. In this sense, the recently developed ICD11 chronic pain classification (see below) is useful for diagnosing complex pain in a hierarchical manner.

\section{Neuromechanical classification}

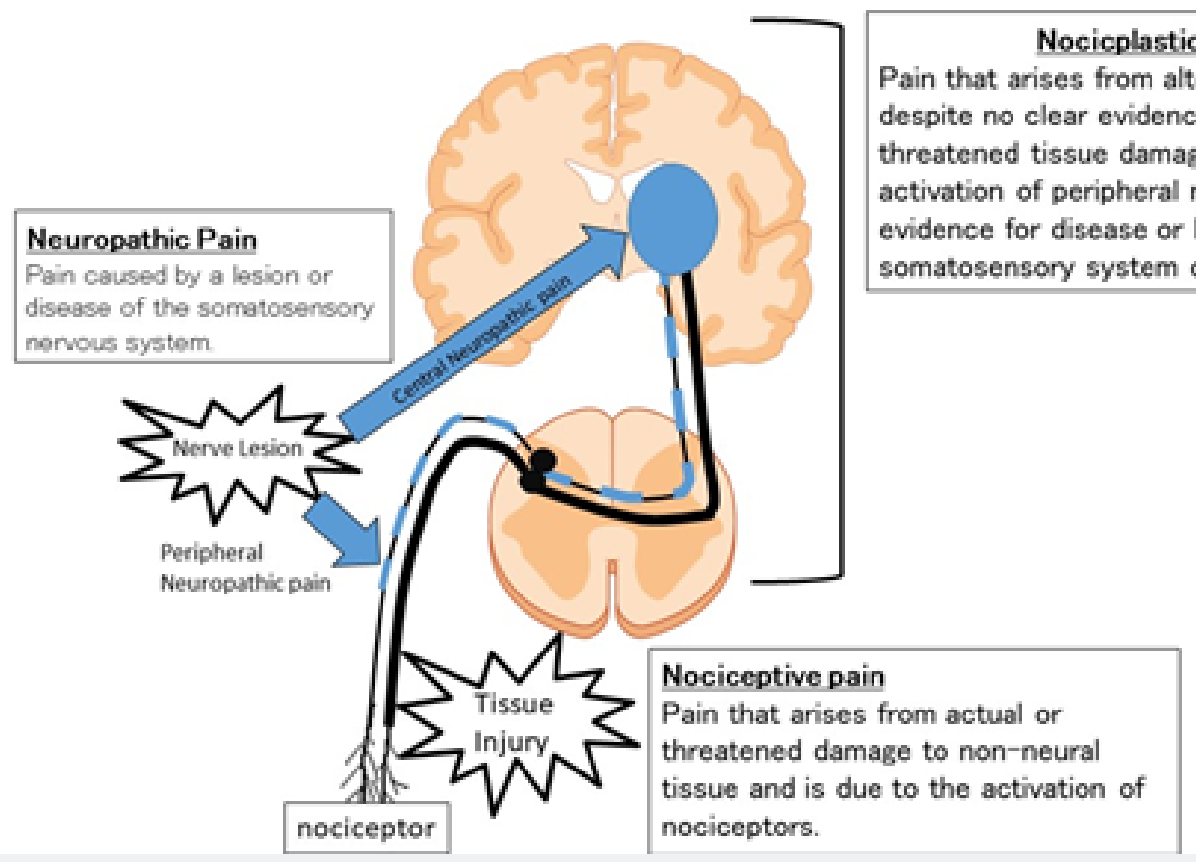

Figure 1: Characteristics of nociceptive, neuropathic, and nociplastic pain. 
Characteristics of nociceptive, neuropathic, and nociplastic pain (Figure 1)

\section{Nociceptive pain}

Nociceptive pain is pain caused by tissue damage, involving. Nociceptive pain is pain caused by the activation of nociceptors located in the distal end of peripheral nerves and by the excitation of peripheral nerves that transmit pain. Most of the pain that we often experience in our daily lives is categorized herein. For example, if a person hits theira toe, he will immediately feel a pinpoint sharp and agile pain (fast pain) in the area where you hit, followed by a sharp but slow pain (slow pain) that lasts for a long time. This is since two different types of nerve fibers (A-delta fibers and C-fibers) activate nociceptors as nociceptive stimuli when strong pressure on the tissue caused by a hard blow is applied. Nociceptive pain also occurs in osteoarthritis. In osteoarthritis, nociceptive pain is caused by repeated physical stress on the inflamed area caused by degenerated tissues, resulting in a condition that cannot be healed, and leadings to a chronic pain situation.

\section{Neuropathic pain}

Neuropathic pain is pain that arises as a direct consequence of a lesion or disease affecting the somatosensory system. For example, pain caused by nerve damage due to stroke, vasculitis, diabetes, genetic abnormalities, infections, chemotherapy, etc.
In particular, central neuropathic pain is caused by damage or disease of the central somatosensory system (spinal cord injury, thalamic pain due to stroke, etc.). Nerve damage causes reactions such as spontaneous pain, excessive response and sensitivity to nociceptor stimulation, and ectopic excitation. These responses are thought to be caused by changes in ion channels that control receptors and signal conduction, as well as abnormalities in the neural network.

\section{Nociceptive Pain [3]}

Nociplastic pain is a pain that arises from altered nociception despite no clear evidence of actual or threatened tissue damage causing the activation of peripheral nociceptors or evidence for disease or lesion of the somatosensory system causing the pain. The term "nociplastic" was coined from "nociceptive plasticity," meaning "plasticity of nociception and pain perception". The pathomechanism of nociplastic pain is not considered simple. Fibromyalgia, the most common type of pain, is often experienced by women and is often triggered by physical as well as psychosocial factors, such as illness or divorce, and is often accompanied by depression. The condition of experiencing high levels of pain is thought to be caused by software (functional) mechanisms rather than hardware mechanisms. Other examples include complex regional pain syndrome, non-specific low back pain, and other disorders.

\section{Classification of chronic pain: International Classification of Diseases ICD-11 $[4,5]$}

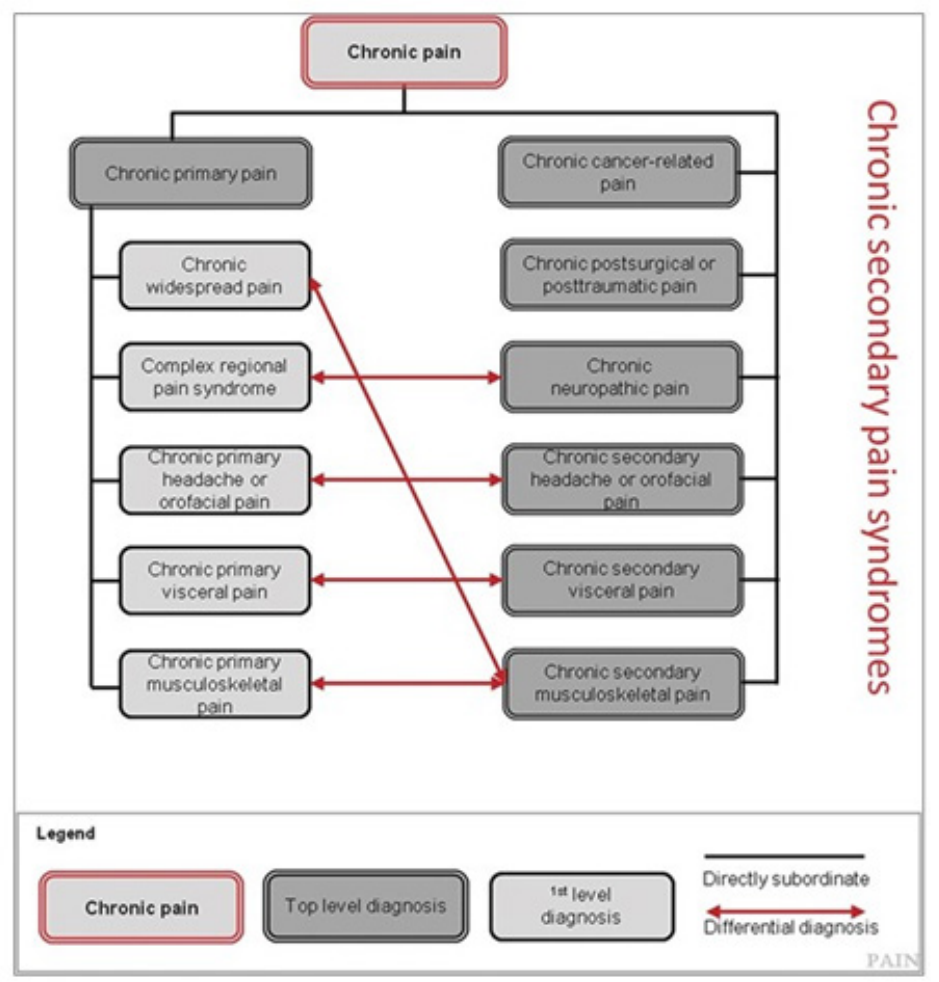

Figure 2: Classification of ICD11 Chronic Pain [6]. 
Chronic pain (chronic pain), as mentioned above, is different from acute pain. Chronic pain is a condition in which pain persists for a certain period (more than three months) and is a complicated condition in which pain develops and persists not only due to organic abnormalities in the musculoskeletal system and nerves but also due to the influence of psychosocial factors and pain behaviors associated with pain. This leads to the fact that it cannot be treated with simple therapies. Until now, chronic pain has not been classified due to its complexity, and various disease names have been used in medical practice based on location and etiology (e.g., sciatica, lumbar disc herniation, etc.). The WHO and IASP have developed a chronic pain classification in ICD11. Chronic pain is divided into two categories: "chronic secondary pain," which has a clear organic cause, and "chronic primary pain," which is a condition in which there is an organic pathology but that alone cannot explain the patient's pain. Chronic secondary pain includes osteoarthritis, diabetic neuropathy, and cancer-related pain. (Figure 2) Chronic secondary pain includes osteoarthritis, diabetic neuropathy, and cancer-related pain, while chronic primary pain includes fibromyalgia, migraine, complex regional pain syndrome, and chronic low back pain. The pain mechanism of chronic primary pain is complex, but it is thought to include the mechanism of nociplastic pain. In the future, it is hoped that pain treatment will become more appropriate by analyzing ICD classification and the effects of treatment.

\section{References}

1. Aydede M (2019) Does the IASP definition of pain need updating? Pain Rep 4(5): e777.

2. Raja SN, Carr DB, Cohen M, Finnerup NB, Flor H, et al. (2020) The revised International Association for the Study of Pain definition of pain: concepts, challenges, and compromises. Pain 161(9): 1976-1982.

3. Aydede M, Shriver A (2018) Recently introduced definition of "nociplastic pain" by the International Association for the Study of Pain needs better formulation. Pain 159(6): 1176-1177.

4. Barke A, Korwisi B, Casser HR, Fors EA, Geber C, et al. (2018) Pilot field testing of the chronic pain classification for ICD-11: the results of ecological coding. BMC Public Health 18(1): 1239.

5. Treede RD, Rief W, Barke A, Aziz Q, Bennett MI, et al. (2019) Chronic pain as a symptom or a disease: the IASP Classification of Chronic Pain for the International Classification of Diseases (ICD-11). Pain 160(1): 19-27.

6. Treede, Rolf-Detlefa, Rief, Winfried, Barke, et al. (2019) Chronic pain as a symptom or a disease: the IASP Classification of Chronic Pain for the International Classification of Diseases (ICD-11). Pain 160(1): 19-27.

\section{Your next submission with Juniper Publishers will reach you the below assets}

- Quality Editorial service

- Swift Peer Review

- Reprints availability

- E-prints Service

- Manuscript Podcast for convenient understanding

- Global attainment for your research

- Manuscript accessibility in different formats

( Pdf, E-pub, Full Text, Audio)

- Unceasing customer service

Track the below URL for one-step submission https://juniperpublishers.com/online-submission.php 